\title{
Modeling of Screw-Dental Structure Interaction: First Step Toward Finite Element Analysis
}

\author{
A. Pasculli* \\ Department of Engineering and Geology, University G. D'Annunzio, \\ Viale dei Vestini 31, 68100 Chieti, ITALY \\ F. Rizzo, M. Mangifesta, and A. Viskovic \\ Department of Engineering and Geology, University G. D'Annunzio, Viale Pindaro 42, 65127 Pescara, ITALY
}

\author{
A. De Sanctis \\ Department of Business Economics, University of Chieti-Pescara, Pescara, ITALY \\ G. Iezzi, V. Perrotti, and A. Piattelli \\ Department of Stomatology and Biotechnological Sciences, \\ University G. D'Annunzio, Viale dei Vestini 31, 68100 Chieti, ITALY \\ G. Aprile \\ Private practice, Rome, ITALY \\ (Received 29 November, 2019)
}

\begin{abstract}
This research is focused on the screw-structure interaction under dynamic impulses aimed to the contact analysis of gap propagation at dental implant-abutment interface under dynamic loading. The purpose is to investigate the fracture mechanism that occurs during the screw penetration in a support and during the support vibration. Numerical Finite Element (FEM) analyses are calibrated through experimental laboratory measurements on support materials and screws typologies. Uncertainty due to the laboratory error propagation is investigated using Polynomial Chaos Expansion (PCE) of experimental measurements. The research is planned through the following steps: FEM calibration trough laboratory experiments on materials; laboratory experiments and material mechanics identification; uncertainty error propagation analysis through PCE expansion; fragility curves for numerical model calibration; FEM models calibration trough laboratory measurements on cycling loads. Therefore, the main purpose of the research activity, of which this article constitutes a preliminary and an explorative step, is to study a methodology that minimizes the local damage of the dental material due to the generation of micro-cracks following the implantation actions. Accordingly, in this paper, preliminary results are reported.
\end{abstract}

AMS Subject Classification: $65 \mathrm{Y} 15$

Keywords: dental material, implant-abutment interface, gap propagation, FEM analysis, microfracture

DOI: https://doi.org/10.33581/1561-4085-2020-23-2-125-129

\section{Introduction}

The study of complex systems generally requires the knowledge of many descriptive

\footnotetext{
${ }^{*}$ E-mail: a.pasculli@unich.it; INDAM Research group GNCS (National Institute of Advanced Mathematics), National Group of Scientific Computing, University G. D'Annunzio, ITALY
}

parameters, not always known with the necessary accuracy. Accordingly, the statistical approach has provided effective tools of scientific investigation in many fields such as windstructures [1, 2], environment [3-6] and also in the field of dental materials. Within this context, the selected method of PCE is an useful and important approach [7], aimed at investigating the uncertainty due to the 
laboratory experiments. Therefore, after having characterized the dental material, which may be considered in all respects as a complex system, the stress state induced by the implantation actions can be studied through the FEM. This type of approach is commonly used in various fields, from structural mechanics [8], to computational fluid dynamics (CFD) $[9,10]$. Furthermore, the FEM has been used also in dental implantology [11, 12].

\section{Materials and methods}

\subsection{Experimental setup}

For this first step, a cylinder, simulating a possible experimental sample, well representative of real dental system, was selected. In Figure 1 a sketch of the facility is reported (the dimension's unit is $\mathrm{mm}$ ).

\subsection{Uncertainty estimation by PCE}

The uncertainty due to the laboratory experiments will be investigated through the PCE. The PCE, is based on a spectral representation of a random variable. On a probability space $\Theta, \Sigma, P$, if one denotes by $\theta$ the random event belonging to the domain $\Theta$, a generic realization of a random variable $\theta$ can be formally written according to Equation 1

$$
V(x, y, z, t ; \theta)=\sum_{j=0}^{p} h_{j}^{*}(\xi(\theta)),
$$

where $V$ is the value of the tension at point $(x, y, z), t$ is time, $P$ the order of the polynomial expansion, $h_{j}^{*}$ Hermite polynomial coefficients; $\psi$ are polynomials of degree $j$ used by the expansion; $\xi$ is the a realization of a seed random variable.

\subsection{Finite element method modelling}

The FEM approach is a standard methodology. In general, the steps to be followed are (1) definition of the problem in term of differential equations; (2) selection of the type and order of FEM to be used in the analysis (in our work, linear interpolation); (3) selection of the mesh for discretization; (4) computation and assemblage of the global matrix; (5) solution of the linear (in our case) or non linear resulting algebraic equation after the application of the most appropriate numerical approach (in our case Galerkin); (6) output the results for the nodal and element variables.

The CAD model of the system was exported to MIDAS computer code [13]. ABAQUS computer code [14], also exploited in [12], will be selected to perform more accurate and complete simulations. Therefore, according to the experimental apparatus that could be realized to obtain values against which to compare the numerical results, a cylinder was discretized by more than 5000 3D axi-symmetrical brick element (Figure 2). The computer code MIDAS, selected as a preliminary tool, is based on the following equations: eq. (2) balance of linear momentum and eq. (3) constitutive relations:

$$
\begin{gathered}
\rho \frac{\partial^{2} u_{i}}{\partial t^{2}}=\frac{\partial^{2} \sigma_{1 i}}{\partial x_{1}^{2}}+\frac{\partial^{2} \sigma_{2 i}}{\partial x_{2}^{2}}+\frac{\partial^{2} \sigma_{3 i}}{\partial x_{3}^{2}}+b_{i}, \quad i=1,2,3 \\
\sigma_{i j}=C_{i j k l}\left(\varepsilon_{k l}-\varepsilon_{k l}^{(0)}\right),
\end{gathered}
$$

where $\rho$ is the material density; $u_{i}$ is the i-th component of the displacement; $\sigma$ is the stresses tensor (Figure 3)

$$
\sigma=\left(\begin{array}{lll}
\sigma_{x x} & \sigma_{x y} & \sigma_{x z} \\
\sigma_{y x} & \sigma_{y y} & \sigma_{y z} \\
\sigma_{z x} & \sigma_{z y} & \sigma_{z z}
\end{array}\right)
$$

and $\epsilon$ is the strains tensor

$$
\varepsilon=\left(\begin{array}{lll}
\varepsilon_{x x} & \varepsilon_{x y} & \varepsilon_{x z} \\
\varepsilon_{y x} & \varepsilon_{y y} & \varepsilon_{y z} \\
\varepsilon_{z x} & \varepsilon_{z y} & \varepsilon_{z z}
\end{array}\right),
$$

$b_{i}$ is the $i$-th component of the external mechanical load; $C_{i j k l}$ are elastic moduli; $\varepsilon_{k l}^{(0)}$ are strains arising from sources other than displacement, for example in the case of heat 


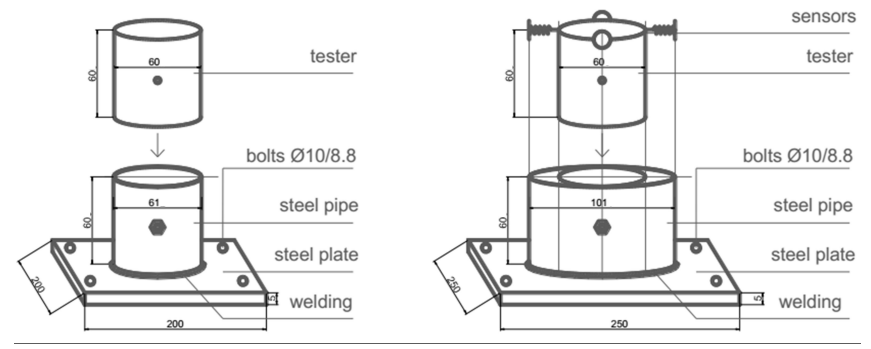

FIG. 1: Sketch of the laboratory facility to be used for experimental measures.

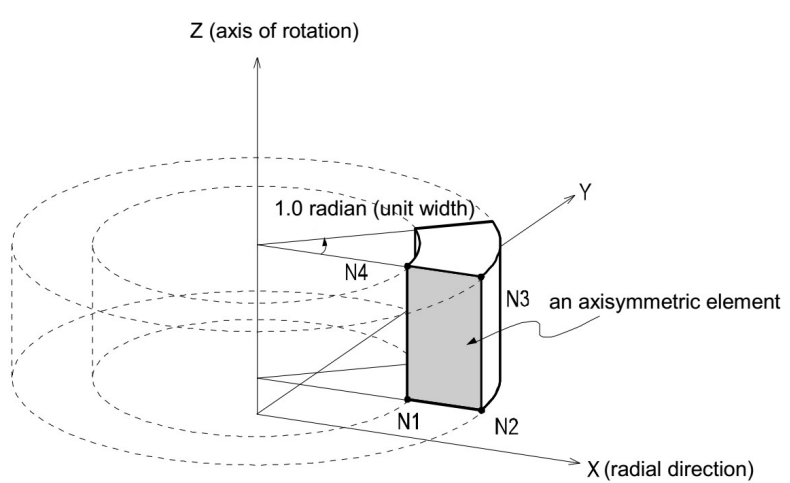

FIG. 2: 3D axi-symmetrical brick element.

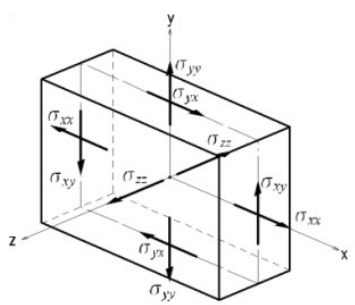

FIG. 3. Sketch of the distribution of each stress tensor components.

production during the screw insertion: $\varepsilon_{k l}^{(0)}=$ $\alpha_{k i}\left(T-T_{0}\right)$ in which $\alpha_{k i}$ are coefficients of linear expansion and $\left(T-T_{0}\right)$ is the temperature increase due to screw action. The rupture conditions are based on combination of the Principal stress components (Invariants), Figure 4. The elements of the grid were assembled according to a concentrated masses scheme using springs and viscous dampers as connections. The solution was selected through an iterative procedure, using a direct integration in the time domain, according to the Newmark scheme

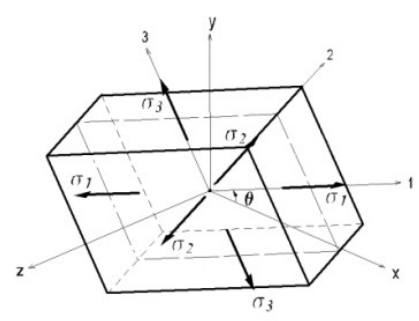

FIG. 4: Sketch of the principal stress components.

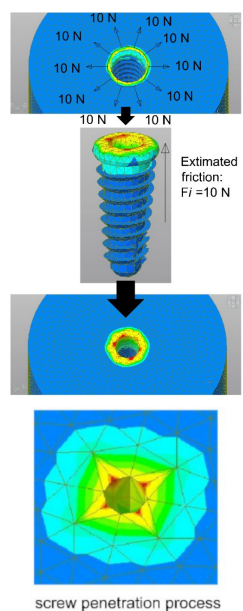

screw penetration process
modelling (quasi-static analyses)

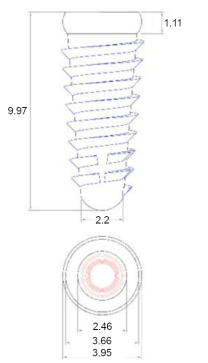

FIG. 5. (color online) MIDAS Numerical realization of the insertion of the screw into the virtual cylindrical sample; visualization of the screw stress state, at the start of the procedure. 
[8]. The calculation procedure uses a system of equations represented in matrix form:

$$
[M] \ddot{u}+[C] \dot{u}+[K] u=R,
$$

where $[M]$ is a mass matrix (lumped mass formulation); $[C]$ is a damping matrix; $[K]$ is a stiffness matrix; $R$ is the load vector.

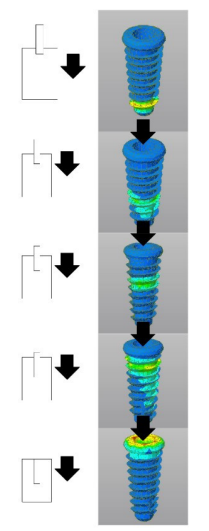

FIG. 6. (color online) MIDAS Numerical realization of the insertion of the screw into the virtual cylindrical sample; visualization of the screw stress state, during insertion.

\section{Results}

As first attempt, aimed at exploring the feasibility of the selected numerical model, the introduction of a screw with a longitudinal load of $10 \mathrm{~N}$ was simulated. In this step, the torque was not included. The scheme of the model and the distribution of the stress state in the virtual specimen are described in Figures 5, 6, 7.

\section{Conclusion}

In this article a first multidisciplinary attempt was presented by our research group to verify the feasibility of a numerical and experimental approach aimed at studying optimization of dental implantation processes. The selected numerical approach was the FEM, which shows the necessary and required flexibility to achieve this purpose, while the probable uncertainties of the system under study could also be managed through the approach to chaotic polynomials and other statistical tools. The ultimate goal is to identify strategies to minimize the impact of the implant on dental and gingival structures to prevent deterioration and microfractures of the affected organic matter, over time.

\section{Acknowledgements}

Special Thanks to engineers Marco Di Giovanni and Chiara Taddeo, for their support to the preliminary FEM analysis.
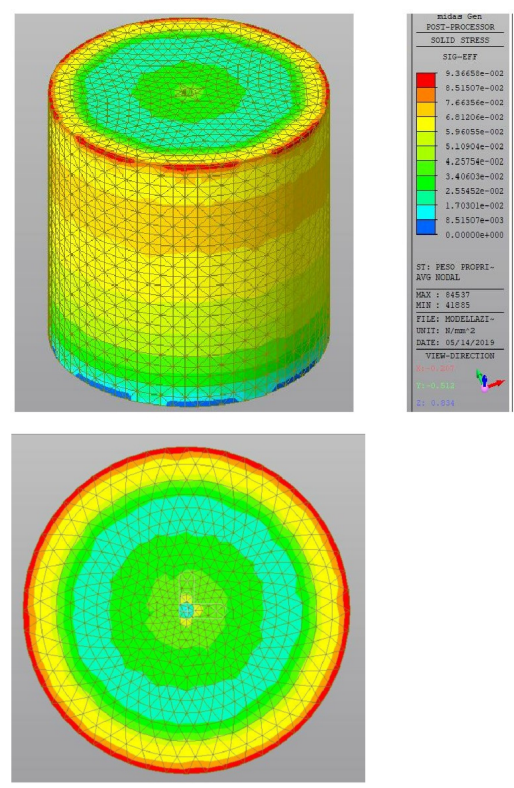

FIG. 7. (color online) MIDAS Numerical realization of the insertion of the screw into the virtual cylindrical sample; visualization of the induced stress state in the virtual cylinder. 


\section{References}

[1] F. Rizzo, L. Caracoglia. Examining wind tunnel errors in scanlan derivatives and flutter speed of a closed-box. J. Wind and Structures 26 (4), 231 (2018).

[2] F. Rizzo, L. Caracoglia, S. Montelpare. Predicting the flutter speed of a pedestrian suspension bridge through examination of laboratory experimental errors. Engineering Structures 172, 589 (2018).

[3] M. Calista, A. Pasculli, N. Sciarra. Reconstruction of the geotechnical model considering random parameters distributions. Engineering Geology for Society and Territory 2: Landslide processes, 1347-1351 (2015).

[4] A. Pasculli, M. Calista, N. Sciarra. Variability of local stress states resulting from the application of Monte Carlo and finite difference methods to the stability study of a selected slope. Engineering Geology 245, 370 (2018).

[5] A. Chiaudani, D. Di Curzio, W. Palmucci, A. Pasculli, M. Polemio, S. Rusi. Statistical and fractal approaches on long time series to surface water/groundwater relationship assessment: A central italy alluvial plain case study. Water $\mathbf{9}, 1$ (2017).

[6] A. Pasculli, S. Palermi, A. Sarra, T. Piacentini, and E. Miccadei, Environmental Modelling and Software 54, 165 (2014).

[7] R. Ghanem and P. Spanos, Stochastic Finite Elements: A Spectral Approach (Springer-Verlag,
New York, 1991).

[8] O. C. Zienkiewicz and R. L. Taylor, Finite Element Method for Solid and Structural Mechanics (Butterworth-Heinemann Elsevier 6th Edition, 2006).

[9] A. Pasculli. CFD-FEM 2D modelling of a local water flow. some numerical results. Alpine and Mediterranean Quaternary. 21, 215 (2008).

[10] A. Pasculli. Viscosity Variability Impact on 2D Laminar and Turbulent Poiseuille Velocity Profiles; Characteristic-Based Split (CBS) Stabilization. In: 5th International Conference on Mathematics and Computers in Sciences and Industry (MCSI). SCOPUS id=2-s2.085070410457, id =2-s2.0-85070410457 (2018).

[11] M. B. Gungor, H. Yilmaz. Evaluation of stress distributions occurring on zirconia and titanium implant supported prostheses: A threedimensional finite element analysis. The Journal of prosthetic dentistry. 346 (2016).

[12] Y. He, A. Fok, C. Aparicio, W. Teng. Contact analysis of gap formation at dental implant abutment interface under oblique loading: A numerical experimental study. Clin.Implant. Dent. Relat. Res. 1 (2019).

[13] MIDAS, Modeling, Integrated Design and Analysis Software, URL http: //www. MidasUser.com .

[14] ABAQUS Dassault Systemes Simulia. Analysis Users Manual, Volume 4; IV:29.3.8-10 (2012). 